\title{
Modulation of the perception of temporal order by attentional and pre-attentional factors
}

\section{H. Haddad Jr., \\ L.R.R. Carreiro \\ and M.V.C. Baldo}

\author{
Departamento de Fisiologia e Biofísica, Instituto de Ciências Biomédicas I, \\ Universidade de São Paulo, São Paulo, SP, Brasil
}

\section{Correspondence \\ M.V.C. Baldo \\ Av. Prof. Lineu Prestes, 1524 \\ Sala 243, ICB I, USP \\ 05508-900 São Paulo, SP \\ Fax: +55-11-3091-7285 \\ E-mail: vinicius@fisio.icb.usp.br \\ Presented at the XVII Annual Meeting of the Federação de Sociedades de Biologia Experimental, Salvador, BA, Brazil, August 28-31, 2002.}

Research supported by FAPESP (grant 96/06618-9 and fellowship 96/06620-3).

Received April 11, 2002 Accepted June 20, 2002

\section{Abstract}

When two stimuli are presented simultaneously to an observer, the perceived temporal order does not always correspond to the actual one. In three experiments we examined how the location and spatial predictability of visual stimuli modulate the perception of temporal order. Thirty-two participants had to report the temporal order of appearance of two visual stimuli. In Experiment 1, both stimuli were presented at the same eccentricity and no perceptual asynchrony between them was found. In Experiment 2, one stimulus was presented close to the fixation point and the other, peripheral, stimulus was presented in separate blocks in two eccentricities $\left(4.8^{\circ}\right.$ and $\left.9.6^{\circ}\right)$. We found that the peripheral stimulus was perceived to be delayed in relation to the central one, with no significant difference between the delays obtained in the two eccentricities. In Experiment 3, using three eccentricities $\left(2.5^{\circ}, 7.3^{\circ}\right.$ and $\left.12.1^{\circ}\right)$ for the presentation of the peripheral stimulus, we compared a condition in which its location was highly predictable with two other conditions in which its location was progressively less predictable. Here, the perception of the peripheral stimulus was also delayed in relation to the central one, with this delay depending on both the eccentricity and predictability of the stimulus. We argue that attentional deployment, manipulated by the spatial predictability of the stimulus, seems to play an important role in the temporal order perception of visual stimuli. Yet, under whichever condition of spatial predictability, basic sensory and attentional processes are unavoidably entangled and both factors must concur to the perception of temporal order.
The judgment of temporal order of a pair of closely timed stimuli is a very old and still unsolved problem (1) that has been attracting the attention of many neurophysiologists and psychologists. When two stimuli are presented to an observer simultaneously or nearly simultaneously, the perceived order does not always correspond to the actual one. Several models of temporal order judg-

\section{Key words}

- Temporal order judgment

- Attention

- Visual perception

- Psychophysics ment have been proposed in the last decades to account for the source of judgment errors (2-5). These models assume that temporal order perception depends on the time when sensory information reaches a central comparator. In the visual system this depends on the transmission latencies of the sensory signals from the retina to the putative central comparator. Indeed, physiological proper- 
ties of the visual pathways will determine the information transmission time.

The idea that some stimuli could receive perceptual priority for further processing led to the notion that there were factors that could alter the time it takes for stimuli to reach awareness. These factors could be divided into pre-attentive and attentive processes. Pre-attentive, or sensory, factors comprise some basic features of the visual stimulus such as its physical intensity $(6,7)$, size $(8,9)$ and location in the visual field $(10)$. Attention is also assumed to influence the speed at which information is transmitted through the visual system $(11,12)$. However, since attention is a modulatory process, only comparisons between two perceptual outcomes, based either on overt behavioral re-

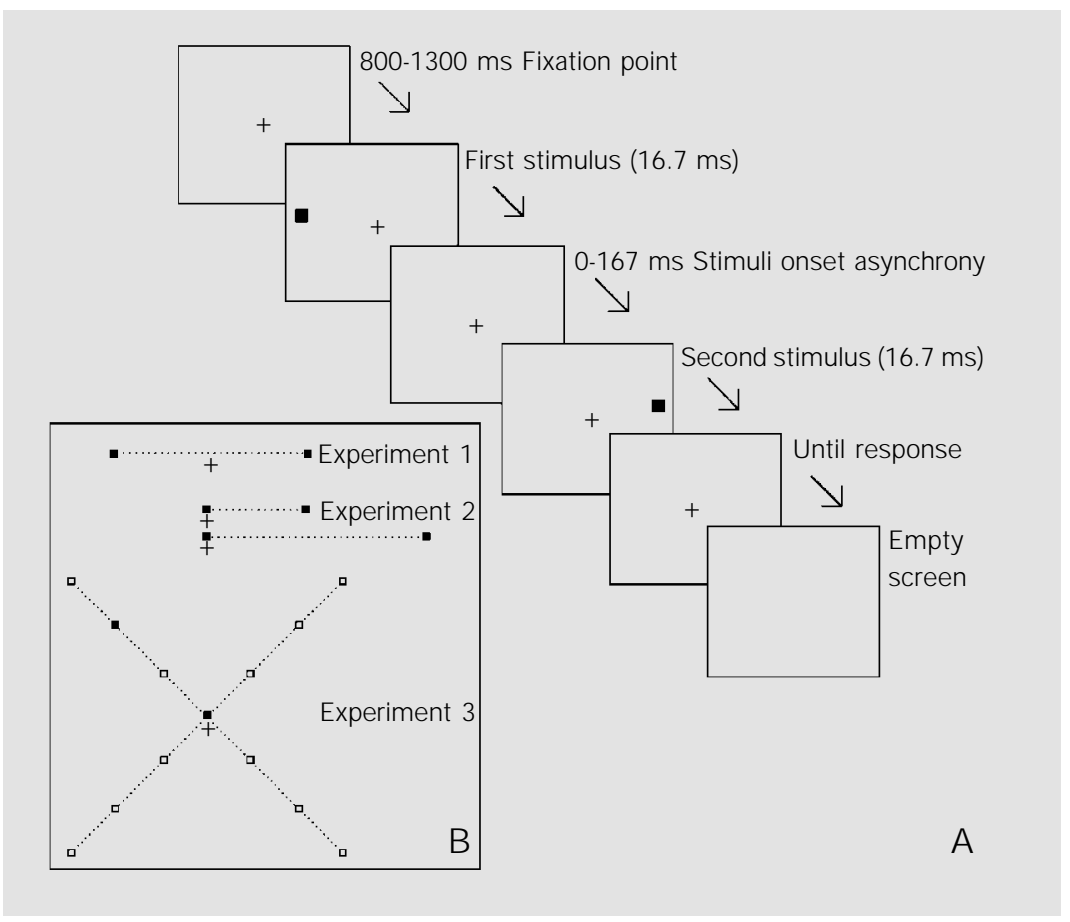

Figure 1. A, Temporal sequence of events used in all three experiments. B, Schematic representation of stimulus display. In Experiment 1, the eccentricity of both stimuli was $4.8^{\circ}$ from the fixation point (the cross in the center of the display). In Experiment 2, the peripheral stimulus was presented in blocked trials in one of two eccentricities $\left(4.8^{\circ}\right.$ and 9.6 $6^{\circ}$. Experiment 3 involved three conditions: 1 ) the peripheral stimulus was presented in the temporal superior visual quadrant, in separate blocks in three different eccentricities $\left(2.5^{\circ}, 7.3^{\circ}\right.$ and $\left.\left.12.1^{\circ}\right), 2\right)$ the peripheral stimulus was randomly presented in the temporal superior visual quadrant, in the same three different eccentricities, and 3) the peripheral stimulus was presented randomly in twelve positions, a combination of the same three eccentricities and four visual quadrants (open squares). sponses or on subjective reports, would reveal the influence of attention (13). Thus, temporal order judgment tasks can provide a reliable psychophysical measure of changes in perceptual latency or in information transmission speed in the visual system (14).

In two experiments we examined in human volunteers how the spatial location of the stimuli in the visual field influences the perception of temporal order. With the aid of a third experiment we were able to investigate the contribution of visual attention to this perceptual task by manipulating the spatial predictability of stimulus presentation. The experimental procedure was reviewed and approved by the Committee on Research Involving Human Subjects, Institute of Biomedical Sciences, University of São Paulo, and informed written consent was obtained from the participants.

In all experiments, participants were seated in a dimly lit and sound-attenuated room. All volunteers had normal or corrected-to-normal vision and were tested monocularly, using their dominant eye, with the contralateral eye being occluded with an ophthalmic patch. Their heads were positioned on a chin and forehead rest at a viewing distance of $57 \mathrm{~cm}$. Participants were required to fixate their gaze (monitored by a video camera) on a point on the center of the monitor screen (fixation point) and report at their own pace, by means of the computer's keyboard, the temporal order of appearance of a pair of stimuli. A two-alternative forcedchoice method was used. The stimulus onset asynchrony was chosen randomly in each trial and with equal probability from time intervals ranging from -167 to $167 \mathrm{~ms}$, in increments of $16.7 \mathrm{~ms}$ (Figure 1A). A session lasted approximately $30 \mathrm{~min}$ and the method of constant stimuli was employed in order to estimate a psychometric function.

Eight volunteers participated in Experiment 1 . In each trial, two brief flashes (two squares subtending $0.12^{\circ}$ of the visual angle) lasting $16.7 \mathrm{~ms}$ each were presented, one 
$4.8^{\circ}$ to the left from the fixation point and the other $4.8^{\circ}$ to the right from the fixation point (Figure 1B). Participants had to report which stimulus was perceived as the first to appear. After each session, a psychometric function was fitted to the data allowing the estimation of the point of subjective equality (PSE). The PSE corresponds to the temporal interval between the two stimuli for which observers have the perception of simultaneity. In Experiment 1 we detected a mean ( \pm SEM) PSE of $0.54 \pm 4.94 \mathrm{~ms}$, which is not statistically different from zero $(\mathrm{P}>0.75)$. Despite the possibility of either sensory differences between temporal and nasal hemifields or a perceptual bias toward left-hand or right-hand stimulus presentation, Experiment 1 was unable to reveal the presence of any significant visual field asymmetry in temporal order perception.

Sensory aspects linked to the morphology and physiology of the retina and visual pathways $(15,16)$ lead to shorter latencies for stimuli occurring in foveal regions than for stimuli occurring peripherally in the visual field. This fact led us to expect that a central stimulus would be perceived prior to a peripheral one. This prediction was tested in Experiment 2. In each trial, two brief flashes (two squares subtending $0.12^{\circ}$ of the visual angle) lasting $16.7 \mathrm{~ms}$ each were presented. One stimulus was always presented close to the fixation point (central stimulus), and the other (peripheral stimulus) was always presented in blocked trials at an eccentricity of either $4.8^{\circ}$ or $9.6^{\circ}$ in the temporal hemifield (Figure 1B). Fourteen participants took part in this experiment.

In Experiment 2 we found a mean PSE that was significantly different from zero $(\mathrm{P}<0.001)$ for both eccentricities of the peripheral stimulus (Figure 2), indicating that the peripheral stimulus had to be presented prior to the central stimulus in order to generate the perception of simultaneity. Oneway ANOVA did not reveal a significant difference between the mean PSE obtained for the two eccentricities tested $\left(\mathrm{F}_{1,13}=0.02\right.$, $\mathrm{P}=0.87$ ). Since the two eccentricities were tested in blocked trials, the participants could predict the location of presentation of the peripheral stimulus, thus being able to allocate previously their attentional focus to that region of the visual field. Therefore, we argue that in this experiment the observed perceptual delay of the peripheral stimulus was caused essentially by sensory (preattentive) mechanisms, supporting the belief that these processes are paramount components of temporal order judgments.

In order to examine how attention possibly modulates these perceptual latencies, we designed Experiment 3, in which the peripheral stimulus was presented with different degrees of spatial predictability. The temporal sequence was identical to that employed in Experiment 2. There were three blocked experimental conditions presented in a balanced order: 1) the peripheral stimulus was presented in separate blocks at eccentricities of $2.5^{\circ}, 7.3^{\circ}$ or $12.1^{\circ}$ in the temporal superior visual quadrant, 2) the peripheral stimulus was randomly presented at one of the same set of visual eccentricities, also in the temporal superior visual quadrant, and 3) the peripheral stimulus was randomly presented at one of twelve locations resulting from the combination of the three eccentricities and the four visual quadrants (Figure 1B). Thus, in condition 1, as also observed in Experi-

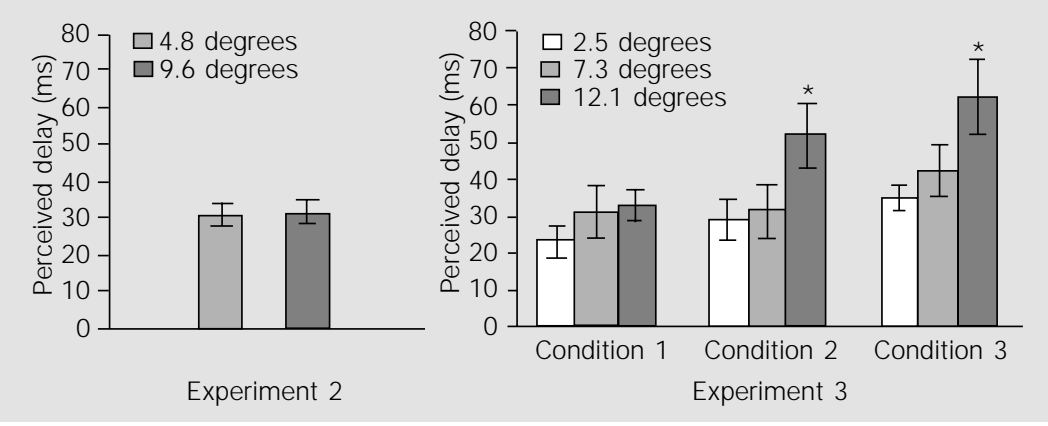

Figure 2. Delay with which the peripheral stimulus was perceived in relation to the central one when both stimuli were presented simultaneously. See legend to Figure 1 for explanation of Experiment 3 conditions. Data are reported as means $\pm \mathrm{SEM}$ in $\mathrm{ms}$. $* \mathrm{P}<0.002$ compared to other eccentricities of the same condition (Tukey HSD test). 
ment 2, participants could predict the location of the peripheral stimulus presentation. In condition 2 the visual quadrant was predictable, but not the peripheral stimulus eccentricity. In condition 3 , the highest unpredictability was assigned to the presentation of the peripheral stimulus. Ten participants took part in this experiment and were tested in all three conditions on different days.

A two-way ANOVA (predictability $\mathrm{x}$ eccentricity) showed that in all three conditions the peripheral stimulus was perceived with a delay in relation to the central one. This perceived delay (Figure 2) increased as either the predictability of the peripheral stimulus was decreased (factor predictability; $\mathrm{F}_{2,18}=7.45, \mathrm{P}<0.005$ ) or its eccentricity was increased (factor eccentricity; $\mathrm{F}_{2,18}=$ 15.82, $\mathrm{P}<0.0001)$. A two-way interaction yielded also a significant effect $\left(\mathrm{F}_{4,36}=3.70\right.$, $\mathrm{P}<0.016$ ). Similarly to Experiment 2, no significant difference was found between the mean PSE measured in the three eccentricities employed in condition 1. However, in conditions 2 and 3 the perceptual delay of the peripheral stimulus was significantly greater in the highest eccentricity in comparison to the less eccentric locations. Since experimental conditions in Experiment 3 were quite similar to each other regarding their sensory features, we are led to believe that the longer delays detected for eccentricity $12.1^{\circ}$ in conditions 2 and 3 are due to the lower spatial predictability assigned to the peripheral stimulus. Although conditions 1 and 2 shared identical settings for stimulus presentation (identical eccentricities, hemifield and visual quadrant), a significant difference between these conditions could be observed regarding the latency of peripheral stimulus (possibly related to predictability differences between these two conditions). Therefore, the presentation of the peripheral stimulus at randomly chosen locations had the effect of precluding the previous allocation of visual attention, leading to perceptual delays that can be thought of as the interaction of sensory and attentional factors.

Basic sensory processes seem to play an important role concerning the temporal order perception of visual stimuli. Yet, the present data show that the modulation of visual latencies is an important consequence of attentional allocation and that these two factors can hardly be disentangled from each other. Further investigation on this topic, already in progress in our laboratory, might possibly shed some light on the interplay between sensory and attentional factors determining the perception of temporal order. Also, we hope that this effort might help us to understand other visual phenomena such as the flash-lag effect (17-19) and the perception of flicker, motion and other rapidly changing visual stimuli $(13,20)$.

\section{Acknowledgments}

We thank Ronald Ranvaud and Luiz Ribeiro-do-Valle for valuable comments and Roberto Vieira for technical assistance.

\section{References}

1. J ames W (1890). The Principles of Psychology. Dover Publications Inc., New York, NY, USA.

2. Stemberg S \& Knoll RL (1973). The perception of temporal order: Fundamental issues and a general model. In: Komblum $S$ (Editor), Attention and Performance IV. Academic Press, New York, NY, USA, 629-685.

3. Allan LG (1975). The relationship between judgments of successiveness and judgments of order. Perception and Psychophysics, 18: 29-36.

4. Ulrich R (1987). Threshold models of temporal-order judgments evaluated by a ternary response task. Perception and Psychophysics, 42: 224-239.

5. J askowski P (1991). Two-stage model for order discrimination. Perception and Psychophysics, 50: 76-82.
6. Roufs J A (1963). Perception lags as a function of stimulus luminance. Vision Research, 3: 81-91.

7. Pins $D \&$ Bonnet C (1996). On the relation between stimulus intensity and processing time: Piéron's law and choice reaction time. Perception and Psychophysics, 58: 390-400.

8. Virsu V \& Hari R (1996). Cortical magnification, scale invariance and visual ecol- 
ogy. Vision Research, 36: 2971-2977.

9. Haddad J r H, Klein S \& Baldo MVC (1999). The contribution of attentional and preattentional mechanisms to the perception of temporal order. In: Taddei-Ferretti C \& Musio C (Editors), Neuronal Bases and Psychological Aspects of Consciousness. World Scientific, Singapore.

10. Carrasco $M$ \& Yeshurun $Y$ (1998). The contribution of covert attention to the setsize and eccentricity effects in visual search. J oumal of Experimental Psychology: Human Perception and Performance, 24: 673-692.

11. Posner MI, Snyder CRR \& Davidson BJ (1980). Attention and the detection of signals. J oumal of Experimental Psychology:
General, 109: 160-174.

12. Driver J (2001). A selective review of selective attention research from the past century. British J oumal of Psychology, 92: 53-78.

13. Hikosaka O, Miyauchi $S \&$ Shimojo $S$ (1993). Focal attention produces illusory temporal order and motion sensation. Vision Research, 33: 1219-1240.

14. Stelmach LB \& Herdman CM (1991). Directed attention and perception of temporal order. J oumal of Experimental Psychology: Human Perception and Performance, 17: 539-550.

15. Masland RH (2001). The fundamental plan of the retina. Nature Neuroscience, 4 : 877-886.
16. Palmer SE (1999). Vision Science. MIT Press, Cambridge, MA, USA.

17. Nijhawan R (1994). Motion extrapolation in catching. Nature, 370: 256-257.

18. Baldo MVC \& Klein SA (1995). Extrapolation or attention shift? Nature, 378: 565566.

19. Baldo MVC, Kihara AH, Namba J \& Klein SA (2002). Evidence for an attentional component or the perceptual misalignment between moving and flashing stimuli. Perception, 31: 17-30.

20. Stelmach LB, Herdman CM \& McNeil KR (1994). Attentional modulation of visual processes in motion perception. J ournal of Experimental Psychology: Human Perception and Performance, 20: 108-121. 